\title{
Predicate Encryption for Secure Remote Storage
}

\author{
Giuseppe Persiano \\ Università di Salerno
}

\begin{abstract}
Predicate encryption is a special encryption method that allows one to release keys to compute specific predicates of the plaintext without having to decrypt. This cryptographic primitive is instrumental for executing search on encrypted data and enables remote storage of data. Predicate encryption dispenses with the need of downloading and decrypting the whole data set whenever a search needs to be performed.

In this talk, the author overviewed security models and constructions proposed and suggested a few applications.
\end{abstract}

